\title{
Câncer colorretal: análise anatomopatológica de 476 colectomias consecutivas em Belo Horizonte (MG)
}

\section{Colorectal cancer: pathologic analysis of 476 consecutive colectomies in Belo Horizonte (MG), Brazil}

\author{
Túlio Pereira Júnior; Antônio José da Cunha Alves²; Ana Margarida M. F. Nogueira³
}

\begin{abstract}
unitermos Carcinoma colorretal Patologia Diagnóstico

\section{resumo}

Introdução: No Brasil, o carcinoma colorretal (CCR) é a terceira causa de óbito por tumores malignos em mulheres e a quinta em homens. Entretanto os dados a respeito deste tumor são escassos. Objetivos: Estudar as características anatomopatológicas do CCR no nosso meio. Materiais e método: Estudaramse 486 casos de CCR diagnosticados consecutivamente segundo protocolo de rotina. As características anatomopatológicas foram analisadas em relação a sexo e idade dos pacientes e topografia do CCR. Resultados: O CCR foi mais freqüente em mulheres $(n=271 ; 56,9 \%)$ que em homens ( $n=205 ; 43,1 \%)$. A média de idade foi de $62,5 \pm 16,04$ anos, e $51(10,7 \%)$ pacientes tinham até 40 anos. Predominou o tipo ulceroinfiltrativo $(n=186 ; 40,8 \%)$, seguido do anular-constritivo $(n=164 ; 36 \%)$. Os tumores do cólon direito foram significativamente maiores do que os do cólon esquerdo $(p<0,001)$. A maioria dos casos foi de adenocarcinomas moderadamente diferenciados ( $n=331 ; 69,8 \%)$; em 105 (23,9\%) havia infiltração de veias, em 232 (52,7\%), de linfáticos e em 82 casos (18,5\%) observou-se infiltração neural. Predominavam tumores em estádio pT3 $(n=308 ; 64,7 \%)$ e pN0 $(n=246 ; 51,7 \%) ; 168(35,3 \%)$ foram Dukes B e 179 (37,6\%), Dukes C. Não foi encontrada relação entre sede do tumor e sexo dos pacientes, tipo histológico e estadiamento. Conclusões: Os dados obtidos foram semelhantes aos da literatura, demonstrando que, em nosso meio, o CCR predomina em mulheres e a maioria dos tumores é detectada em fase avançada da doença.
\end{abstract}

Background: In Brazil, colorectal carcinoma (CRC) is the third cause of death by malignant tumors among women and the fifth among men. Objectives: To analyze the pathologic characteristics of CRC in Brazilian patients. Material and Methods: 486 CRC diagnosed consecutively were studied. Pathologic characteristics were analyzed according to the patients' gender and age and tumor location. Results: CRC was more frequent in women ( $\mathrm{n}=$ $271 ; 56.9 \%)$ than in men $(\mathrm{n}=205 ; 43.1 \%)$. The median age was $62.5 \pm 16.04$ years and $51(10.7 \%)$ were younger than 41 years. Ulcer-infiltrative tumors were more common $(\mathrm{n}=186 ; 40.8 \%)$ followed by annularconstrictive ones $(n=164 ; 36.0 \%)$. Tumors raised at the right colon were significantly larger than those arising in the left colon $(\mathrm{p}<0,001)$. Most cases were moderately differentiated adenocarcinomas ( $\mathrm{n}=331 ; 69.8 \%)$, lymphatic vessels were invaded in 232 (52.7\%), veins in 105 (23.9\%) and nerves in 82 (18.5\%). pT3 ( $\mathrm{n}=308$; $64.7 \%)$ and $p N O(n=246 ; 51.7 \%)$ were predominant; 168 (35.3\%) CRC were Dukes B stage and 179 (37.6\%) were Dukes $C$. No association was found between tumor location and patients' gender, tumor histological type or stage. Conclusions: Our data are similar to those on record. They show that among our patients CRC occurs predominantly in women and most tumors are detected in an advanced stage. key words

Colorectal carcinoma

Pathology

Diagnosis

. Bolsista de iniciação científica da Fundação de Amparo à Pesquisa do Estado de Minas Cerais (FAPEMIC).

2. Bolsista de iniciação cientifica do Conselho Nacional de Desenvolvimento Científico e Tecnológico (CNPq).

3. Doutora; professora-adjunta; bolsista do CNPq.

Departamento de Anatomia Patológica e Medicina Legal da Faculdade de Medicina da Universidade Federal de Minas Cerais (UFMG). 


\section{Introdução}

O carcinoma colorretal (CCR) apresenta ampla variação de freqüência em todo o mundo e sua incidência está aumentando nos países industrializados ${ }^{(9,12,13,25,26)}$. No Brasil, o CCR é a terceira causa de óbito por tumores malignos em mulheres e a quinta em homens ${ }^{(12)}$. Geralmente o diagnóstico da doença é feito em fase avançada, comprometendo assim o prognóstico e a sobrevida dos pacientes. Por este motivo, além do sistema de estadiamento TNM(29), a pesquisa de marcadores de prognóstico tem sido, mais recentemente, objeto de estudo $(2,3,18,24)$.

As características anatomopatológicas do tumor são importantes para o estadiamento ${ }^{(1,8,5)}$ e a definição da terapêutica a ser empregada ${ }^{(17)}$. O nível de infiltração da parede do órgão, assim como invasão de veias, acometimento de nervos ${ }^{(7,23)}$ e metástases linfonodais(20) ou em órgãos à distância, está intimamente relacionado com pior prognóstico da doença ${ }^{(5,17)}$. O estadiamento convencional é feito pelas classificações de Dukes ${ }^{(8)}$ e Astler-Coller ${ }^{(1)} \mathrm{e}$ $\mathrm{TNM}^{(29)}$, que vem sendo cada vez mais utilizada.

O CCR é atualmente uma das neoplasias mais freqüentemente examinadas no nosso serviço, o que motivou a utilização de protocolo padronizado a partir de $1998^{(29)}$. O objetivo deste trabalho foi avaliar as características anatomopatológicas do CCR de uma série consecutiva de casos de pacientes operados no nosso hospital.

\section{Material e método}

Estudaram-se CCRs de pacientes operados no Hospital das Clínicas da Universidade Federal de Minas Gerais (HC/ UFMG), de janeiro de 1993 a dezembro de 2003. As peças cirúrgicas foram analisadas no Serviço de Anatomia Patológica do Departamento de Anatomia Patológica e Medicina Legal da Faculdade de Medicina da UFMG.

Os dados foram levantados a partir das requisições dos exames e laudos anatomopatológicos padronizados através da utilização de protocolo individual ${ }^{(28)}$ (Anexo). As lâminas e os prontuários dos pacientes foram revistos quando antecediam a data de utilização do protocolo.

As variáveis estudadas foram sexo, idade dos pacientes, topografia da lesão, classificação macroscópica, dimensões do tumor, classificação histológica de acordo com a Organização Mundial da Saúde (OMS)(11), resposta linfocítica peritumoral, invasão de veias e nervos e estadiamento $\mathrm{TNM}^{(29)}$. Os pacientes foram divididos de acordo com a idade, em dois grupos, até 40 anos e acima desta idade, ou estratificados por décadas. Quanto à topografia, os casos foram agrupados em CCR do cólon direito, desde o ceco até o cólon transverso, e do cólon esquerdo, da flexura esplênica ao reto. Tumores localizados na transição sigmóide/reto foram topografados como do retossigmóide.

As características anatomopatológicas dos tumores foram analisadas em relação a sexo e idade dos pacientes e localização da lesão. Compararam-se as características do tumor nos diversos grupos etários. No que diz respeito à topografia, analisaram-se comparativamente as características dos tumores em cada segmento colônico e nos cólons direito e esquerdo. Finalmente, analisou-se a incidência de CCR no cólon direito e no esquerdo ao longo do período de 1993 a 2003.

Os casos cujos tumores tinham sítio primário desconhecido e aqueles que sofreram remissão após terapêutica neo-adjuvante foram excluídos do estudo. Não foi possível a análise de todas as variáveis em todos os casos por problemas inerentes à amostra.

O estudo foi aprovado pelo Comitê de Ética em Pesquisa de UFMG (Processo no 327/03).

\section{Resultados}

A amostra inicial foi composta por 486 peças cirúrgicas de colectomias, das quais foram excluídas dez devido à terapia neo-adjuvante. Portanto, o estudo compreendeu a análise de 476 casos.

Notou-se discreta prevalência de pacientes do sexo feminino $(n=271 ; 56,9 \%)$ em relação ao masculino $(n=$ $205 ; 43,1 \%)$, e a média de idade foi de $62,5 \pm 16,04$ anos, com variação de 20 a 103 anos, sem diferença quanto ao sexo.

A maioria dos CCRs foi diagnosticada em pacientes entre 60 e 80 anos (Figura 1). Não houve diferença estatisticamente significativa entre os grupos de pacientes até 40 anos ( $n=51 ; 10,7 \%)$ e acima desta idade $(n=423 ; 89,3 \%)$ no que diz respeito a sexo, localização, aspecto macroscópico, tipo histológico, resposta linfocítica no tecido peritumoral, invasão de estruturas e estadiamento $(p>0,05)$. Houve maior freqüência de tumores associados a polipose adenomatosa no grupo de pacientes jovens $(3 / 51 ; 6 \%)$ do que no de mais velhos $(2 / 423 ; 0,5 \%)(p=0,01)$.

Com relação à localização do tumor houve predomínio do reto $(n=129 ; 37,1 \%)$, seguindo-se sigmóide $(n=98$; $20,6 \%)$ e retossigmóide $(n=49 ; 10,3 \%)$. Quando se agruparam os tumores em relação aos colons direito e esquerdo, 
notou-se prevalência deste último com $322(68,6 \%)$ casos diagnosticados (Figura 2).

O padrão macroscópico predominante foi ulceroinfiltrativo ( $n=186 ; 40,8 \%$ ), seguindo-se os tipos anularconstritivo $(n=164 ; 36 \%)$, polipóide $(n=77 ; 16,9 \%)$ e em placa $(n=29 ; 6,4 \%)$.

Os tumores tinham volume médio de $78,2 \pm 126,4 \mathrm{~cm}^{3}$, sendo os maiores localizados no ceco $\left(115,4 \pm 122,6 \mathrm{~cm}^{3}\right)$ e os menores, na flexura esplênica $\left(15,9 \pm 16,7 \mathrm{~cm}^{3}\right)$. Geralmente, os CCRs localizados no cólon direito foram significativamente maiores, em todas as suas dimensões $(p<$ $0,02)$, que os localizados no cólon esquerdo (Tabela 1 ).

A maioria dos tumores correspondeu a adenocarcinomas ( $n=411 ; 86,3 \%)$, moderadamente $(n=331 ; 69,8 \%)$ ou bem diferenciados ( $n=107 ; 22,6 \%$ ). Tumores pouco diferenciados ou indiferenciados foram bem menos freqüentes $(n=24 ; 5,1 \%)$. Diagnosticaram-se $45(9,5 \%)$ carcinomas mucinosos (colóides) e dez (2,1\%) carcinomas de células em anel de sinete. Os restantes corresponderam a tipos mais raros: quatro adenoescamosos $(0,8 \%)$, três de pequenas células $(0,6 \%)$ e três de células escamosas $(0,6 \%)$.

Observou-se associação entre o tipo histológico, a localização e o comportamento biológico. O adenocarcinoma foi significativamente mais freqüente no cólon esquerdo ( $n=289 ; 70,8 \%)$ do que no direito $(n=119 ; 29,2 \%)$, quando em comparação com o adenocarcinoma mucinoso, que acometia igualmente os colons direito $(n=26 ; 48,1 \%)$ e esquerdo $(n=28 ; 51,9 \%)(p=0,007)$. Os CCRs colóides acometiam linfonodos mais freqüentemente $(p=0,04)$ e apresentavam margens cirúrgicas comprometidas $(p=$ $0,01)$, mesmo não havendo diferença quanto à infiltração da parede do órgão (pT) ou em outros indicadores histopatológicos de agressividade tumoral.

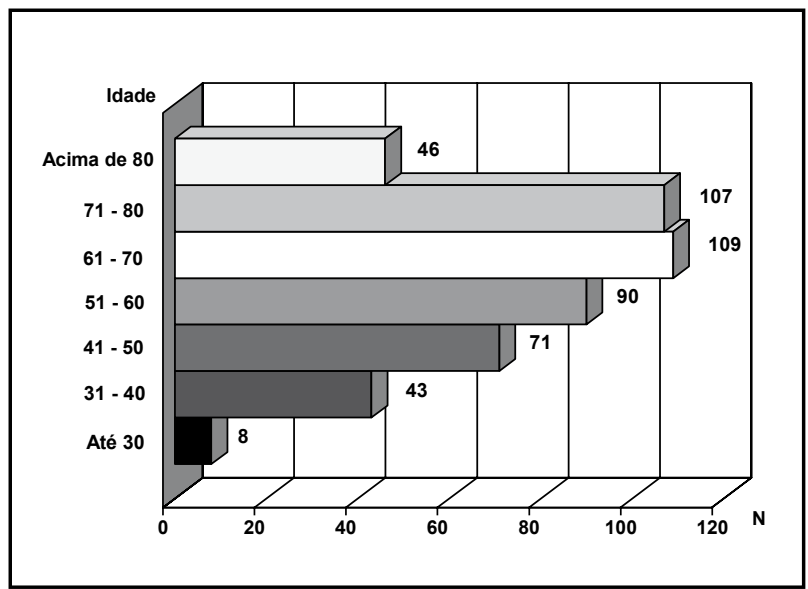

Figura 1 - Distribuição de carcinoma colorretal segundo a idade dos pacientes, estratificada por décadas

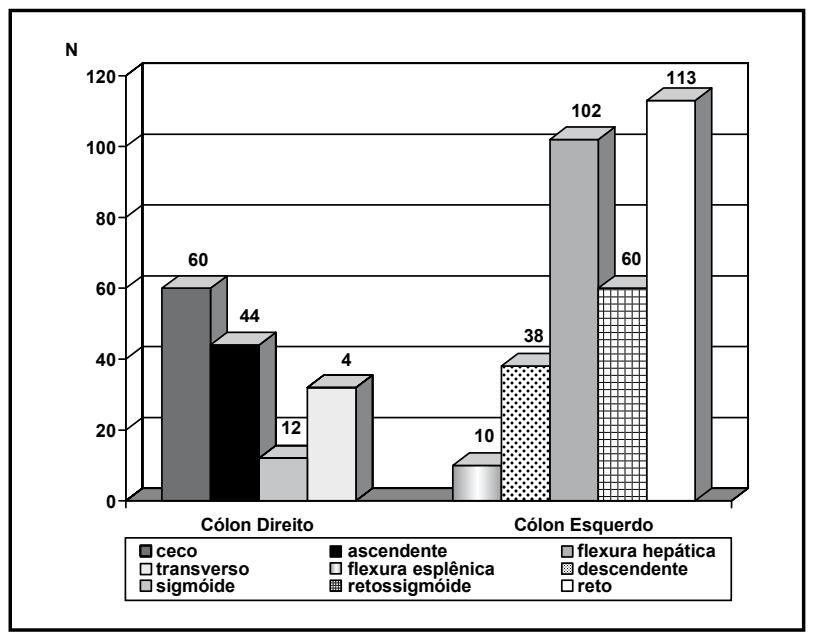

Figura 2 - Distribuição de 476 carcinomas colorretais quanto à localização

Tabela 1

\section{Dimensōes de 476 carcinomas colorretais de acordo com a localização}

\begin{tabular}{lcccc}
\hline & Volume & Comprimento & Largura & Espessura \\
Cólon direito* & 101,3 & 6,7 & 5,5 & 2,8 \\
Ceco & 115,4 & 6,9 & 6,5 & 2,9 \\
Ascendente & 97,4 & 6,1 & 4,8 & 2,8 \\
Flexura hepática & 87,7 & 6,5 & 5,1 & 2 \\
Transverso & 79,1 & 7,1 & 4,6 & 2,8 \\
Cólon esquerdo* & 67,4 & 5,1 & 4,1 & 2,5 \\
Flexura esplênica & 15,9 & 3,8 & 2,5 & 1,4 \\
Descendente & 94,6 & 5,7 & 4,4 & 3,5 \\
Sigmóide & 98,9 & 5,4 & 4,4 & 3,4 \\
Retossigmóide & 46,3 & 5,2 & 4,1 & 2,3 \\
Reto & 51,1 & 4,9 & 3,9 & 1,7 \\
\hline
\end{tabular}

${ }^{*} p<0,001$ 
Em 105 casos (23,9\%) havia infiltração de veias, em 232 (52,7\%), de linfáticos, e em 82 casos (18,5\%) observou-se invasão de nervos. A resposta linfocítica peritumoral foi discreta na maior parte das vezes.

Quanto ao comportamento biológico do CCR, a maioria dos casos foi classificada como pT3 $(n=308 ; 64,7 \%)$ (Figura 3), não se identificando metástases linfonodais na maioria dos casos que foram classificados como pNo $(n=$ $159 ; 33,4 \%)$ ou $\mathrm{pNx}(n=187 ; 39,3 \%)$ quando se dissecaram menos de 12 linfonodos (Figura 4).

Os tumores tinham características semelhantes tanto em homens como em mulheres, sem diferença em relação à idade média (Tabela 2) ou estratificada por décadas $(p>0,05)$.

Não se observou diferença estatisticamente significativa quanto às características do CCR em relação à localização (Tabela 3). A proporção de CCR nos colons direito e es- querdo manteve-se idêntica ao longo do período estudado (Figura 5).

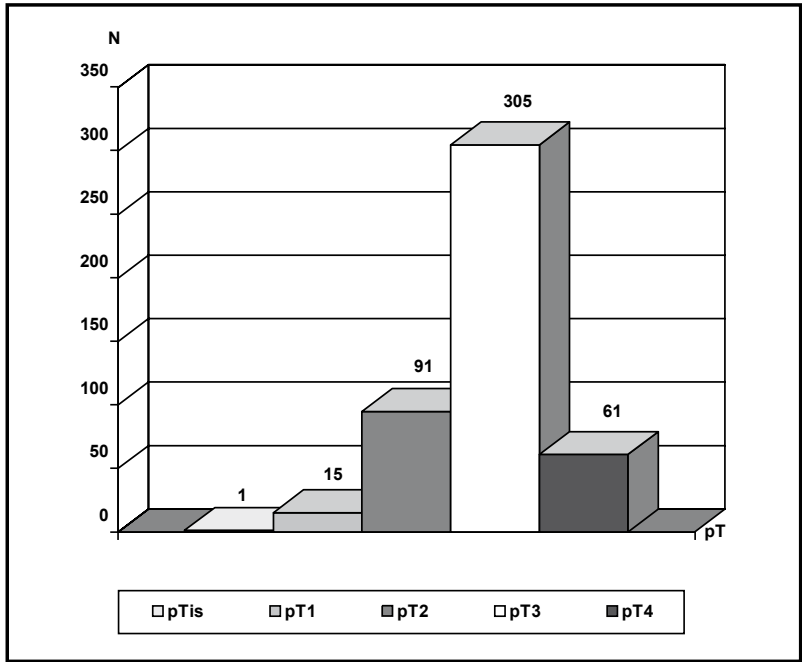

Figura 3 - Distribuição de 476 casos de carcinoma colorretal em relação à profundidade do tumor (pT) segundo o sistema TNM

\section{Tabela 2}

\section{Caracteristicas de $\mathbf{4 7 6}$ carcinomas colorretais em homens e mulheres}

\begin{tabular}{lcc}
\hline Características dos tumores & Homens & Mulheres \\
Idade \pm dp & $61,3 \pm 16,04$ & $61,48 \pm 16,04$ \\
Cólon direito & & \\
$\quad$ Ceco & $21(9,4 \%)$ & $38(14,1 \%)$ \\
Ascendente & $19(10,4 \%)$ & $25(9,3 \%)$ \\
Flexura hepática & $5(2,5 \%)$ & $7(2,6 \%)$ \\
Transverso & $14(6,9 \%)$ & $18(6,7 \%)$ \\
Cólon esquerdo & & \\
Flexura esplênica & $5(2,5 \%)$ & $5(1,9 \%)$ \\
Descendente & $14(6,9 \%)$ & $24(8,9 \%)$ \\
Sigmóide & $39(19,3 \%)$ & $59(21,9 \%)$ \\
Retossigmóide & $21(10,4 \%)$ & $28(10,4 \%)$ \\
Reto & $64(31,7 \%)$ & $65(24,2 \%)$ \\
Volume médio & $85,84 \mathrm{~cm} 3$ & $72,32 \mathrm{~cm} 3$ \\
Infiltração de veias & $49(25,5 \%)$ & $57(23,1 \%)$ \\
Infiltração de linfáticos & $103(53,4 \%)$ & $130(52,8 \%)$ \\
Infiltração de nervos & $40(20,8 \%)$ & $45(18 \%)$ \\
pT & $1(0,5 \%)$ & \\
pTis & $7(3,4 \%)$ & $0(0 \%)$ \\
pT1 & $40(19,5 \%)$ & $8(3 \%)$ \\
pT2 & $132(64,4 \%)$ & $53(19,6 \%)$ \\
pT3 & $25(12,2 \%)$ & $176(64,9 \%)$ \\
pT4 & & $34(12,5 \%)$ \\
pN & $114(55,6 \%)$ & \\
pN0 & $26(12,7 \%)$ & $132(48,7 \%)$ \\
pN1 & $37(18 \%)$ & $39(14,4 \%)$ \\
pN2 & $28(13,7 \%)$ & $46(17 \%)$ \\
pNx & & $54(19,9 \%)$ \\
\hline & & \\
\hline & & \\
& & \\
& &
\end{tabular}




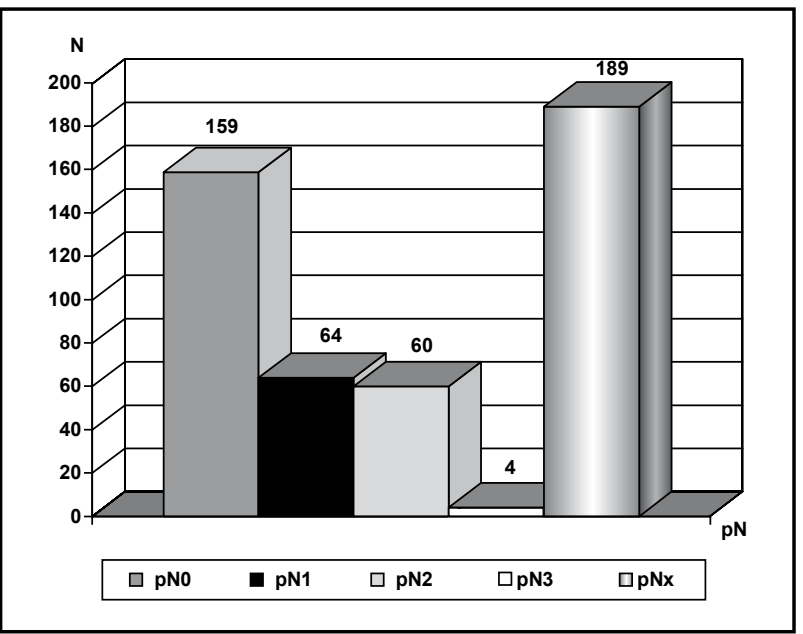

Figura 4 - Classificação da presença de metástase linfonodal $(\mathrm{pN})$ segundo 0 sistema TNM em 476 casos de carcinoma colorretal

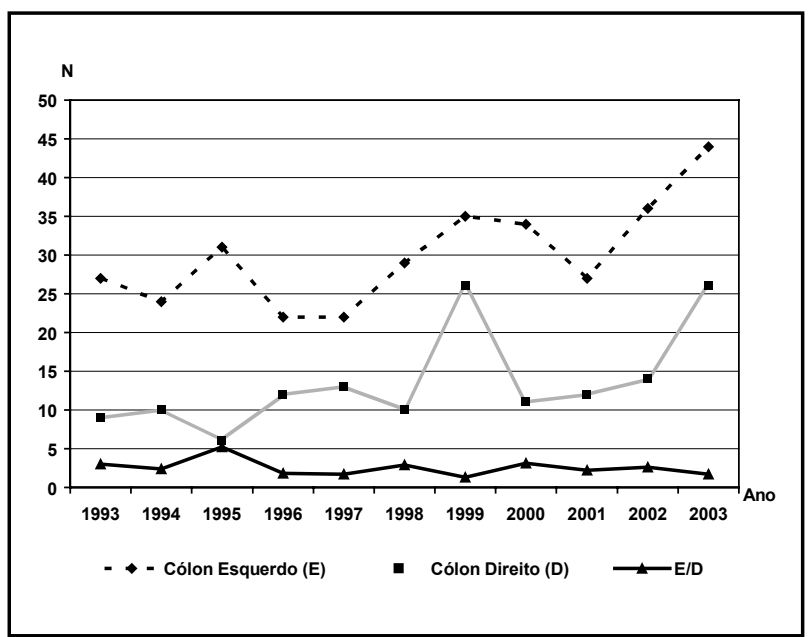

Figura 5 - Incidência de carcinoma colorretal nos colons direito e esquerdo ao longo do período estudado (1993-2003)

\section{Tabela 3 Características de 476 CCR com relação à localizaçăo}

\begin{tabular}{lcc}
\hline & Cólon direito & Cólon esquerdo \\
Idade & $60,72 \pm 14,71$ & $63,07 \pm 15,97$ \\
Sexo & & \\
$\quad$ Masculino & $141(43,8 \%)$ & $61(40,9 \%)$ \\
Feminino & $181(56,2 \%)$ & $88(59,1 \%)$ \\
Volume médio & $67,583 \mathrm{~cm}^{3}$ & $10,28 \mathrm{~cm}^{3}$ \\
Infiltração de linfáticos & $158(53,4 \%)$ & $72(52,2 \%)$ \\
Infiltração de nervos & $60(20,2 \%)$ & $24(17 \%)$ \\
Infiltração de veias & $75(25,4 \%)$ & $29(20,9 \%)$ \\
pT & & \\
pTis & $1(0,3 \%)$ & $0(0 \%)$ \\
pT1 & $9(2,8 \%)$ & $5(3,4 \%)$ \\
pT2 & $73(22,7 \%)$ & $18(12,2 \%)$ \\
pT3 & $194(60,2 \%)$ & $108(73 \%)$ \\
pT4 & $45(14 \%)$ & $15(10,1 \%)$ \\
pN & & \\
pN0 & $95(29,5 \%)$ & $62(41,6 \%)$ \\
pN1 & $44(13,7 \%)$ & $20(13,4 \%)$ \\
pN2 & $36(11,2 \%)$ & $24(16,1 \%)$ \\
pN3 & $3(0,9 \%)$ & $1(0,7 \%)$ \\
pNx & $144(44,7 \%)$ & $40(26,8 \%)$ \\
\hline
\end{tabular}

Em 106 de 284 (37,3\%) casos identificaram-se adenomas em correspondência com o tumor invasor. Estes casos apresentavam uma incidência maior de tumores sincrônicos $(p=0,007)$, infiltravam a parede do órgão menos profundamente $(p<0,001)$, o que correspondia a melhor estadiamento, e tinham menor incidência de invasão de veias $(p=0,001)$ e de nervos $(p=0,01)$. Entretanto, quando se comparou a invasão de vasos linfáticos ou linfonodos com a presença de adenoma, não se observou diferença estatisticamente significativa (Tabela 4).

Tumores sincrônicos foram encontrados em 20 (4,2\%) pacientes, dos quais quatro $(0,8 \%)$ apresentavam pelo menos dois tumores. Destes, dez (50\%) se localizavam no cólon direito e seis (30\%) no ceco. No estudo destes tumores não foi encontrada diferença estatisticamente significativa quanto a estadiamento ou critérios de agressividade quando em comparação com tumores únicos $(p>0,05)$. 
Tabela 4

Características anatomopatológicas do carcinoma colorretal associadas à presença de adenoma

\begin{tabular}{|c|c|c|c|}
\hline \multirow[t]{2}{*}{ Características anatomopatológicas } & \multicolumn{2}{|c|}{ Adenoma } & \multirow[b]{2}{*}{ Valor de $p$} \\
\hline & Sim & Não & \\
\hline \multicolumn{4}{|l|}{ Invasão de veias } \\
\hline Sim & $47(27,2 \%)$ & $13(13,5 \%)$ & \multirow[t]{2}{*}{0,02} \\
\hline Não & $126(72,8 \%)$ & $83(86,5 \%)$ & \\
\hline \multicolumn{4}{|l|}{ Invasão de linfáticos } \\
\hline Sim & $80(46,2 \%)$ & $45(45,9 \%)$ & \multirow[t]{2}{*}{0,9} \\
\hline Não & $93(53,8 \%)$ & $53(54,1 \%)$ & \\
\hline \multicolumn{4}{|l|}{ Invasão de nervos } \\
\hline Sim & $44(25,4 \%)$ & $9(9,2 \%)$ & \multirow[t]{2}{*}{0,002} \\
\hline Não & $129(74,6 \%)$ & $89(90,8 \%)$ & \\
\hline \multicolumn{4}{|l|}{ Metástase linfonodal } \\
\hline $\operatorname{Sim}$ & $38(36,2 \%)$ & $79(44,4 \%)$ & \multirow[t]{2}{*}{0,2} \\
\hline Não & $67(63,8 \%)$ & $99(55,6 \%)$ & \\
\hline \multicolumn{4}{|c|}{ Comprometimento de serosa (pT3 e pT4) } \\
\hline Sim & $137(77,8 \%)$ & $60(58,8 \%)$ & \multirow[t]{2}{*}{0,002} \\
\hline Não & $39(22,2 \%)$ & $42(41,2 \%)$ & \\
\hline \multicolumn{4}{|l|}{ Lesões sincrônicas } \\
\hline Sim & $4(2,2 \%)$ & $11(10,4 \%)$ & \multirow[t]{2}{*}{0,007} \\
\hline Não & $174(97,7 \%)$ & $94(89,5 \%)$ & \\
\hline
\end{tabular}

\section{Discussão}

A análise de 476 pacientes mostrou prevalência relevante do sexo feminino, perfil concordante com os dados disponibilizados pelo Instituto Nacional do Câncer (INCa) ${ }^{(12)}$, sendo escassa a literatura nacional para comparação. A população mais afetada tinha entre 50 e 75 anos, sendo a idade média de 62 anos, semelhante à encontrada na literatura $(7,13,17,24,25)$, mostrando prevalência da doença na população mais velha. Por outro lado, é de ressaltar, na nossa casuística, o elevado número de pacientes com menos de 41 anos, que representavam 10,7\% da amostra. Mendes et al. ${ }^{(17)}$ mostraram incidência semelhante, de $13,1 \%$, de pacientes com menos de 40 anos. O CCR em pacientes jovens (até 40 anos) foi descrito como mais avançado, mais volumoso e com indicadores histopatológicos de maior grau de agressividade, como pouca diferenciação, invasão de vasos linfáticos, sangüíneos e nervos, além de uma maior incidência de tumores mucossecretores ${ }^{(6,17)}$. Na análise de nossa casuística não encontramos qualquer relação entre características dos tumores e idade dos pacientes, mesmo considerando os grupos até 40 anos e acima desta idade. Em pacientes jovens é importante investigar associações com síndromes genéticas, como câncer colorretal heredi- tário sem polipose familiar (síndrome de Lynch) e polipose adenomatosa familiar, selecionando candidatos a testes genéticos para determinação do caráter hereditário da doença ${ }^{(4,6,16,22,27)}$. Esta análise será feita futuramente em nossos casos. Tem sido chamada atenção para a presença ou não de instabilidade de microssatélites, que estaria relacionada com tumores mucinosos, menor freqüência de invasão de vasos, menor número de metástases linfonodais e melhor prognóstico, do que aqueles tumores sem instabilidade ${ }^{(24)}$. $\mathrm{Na}$ nossa análise apenas foi verificada maior incidência de tumores relacionados a polipose adenomatosa, não havendo diferença significativa entre as outras variáveis analisadas.

A literatura tem apontado para um aumento da incidência de tumores no cólon direito nos últimos anos ${ }^{(10,25)}$. Segundo alguns autores, justifica-se a distribuição topográfica porque o cólon tem características embriológicas e biológicas diferentes, podendo estar envolvidas na patogênese do CCR por mecanismos distintos e, conseqüentemente, comportamento biológico diferente ${ }^{(10)}$. O rastreamento e o tratamento dos adenomas colônicos mais freqüentes no cólon esquerdo podem estar relacionados com o aumento relativo de incidência de CCR no cólon direito observado 
recentemente. Na nossa série, notou-se um predomínio de tumores no cólon esquerdo, principalmente sigmóide e reto, semelhante ao relatado na literatura $(7,10,17,25)$. A comparação da incidência de tumores do cólon esquerdo com o direito, ano a ano, não apresentou variação significativa no período.

O CCR apresentou variação de volume relacionada com a sua localização. No presente estudo houve diferença significativa entre todas as dimensões dos tumores do cólon direito (maiores) e esquerdo (menores). Esta diferença não foi acompanhada de piora dos critérios histopatológicos de prognóstico ou do estadiamento, discordando de outros trabalhos ${ }^{(25,27)}$, que mostraram pior estadiamento para os tumores do cólon esquerdo.

Observamos que em apenas $3,4 \%$ dos pacientes 0 tumor se infiltrava até a submucosa. De acordo com a classificação de Dukes ${ }^{(8)}$, apenas $15 \%$ dos pacientes apresentava a doença em estágio inicial (Dukes, A). Os $85 \%$ restantes tinham doença em estágio avançado, o que correspondeu a um prognóstico mais reservado ${ }^{(1,5,8,23,26)}$.

A invasão de nervos encontrada em $18,5 \%$ dos pacientes, de veias em $23,5 \%$ e de linfáticos em $52,7 \%$ foi associada a pior prognóstico ${ }^{(7,23)}$. A freqüência de invasão dessas estruturas foi semelhante ao que se encontra na literatura ${ }^{(7)}$.

Na nossa casuística os tumores mucinosos tinham pior estadiamento linfonodal e maior incidência de casos com margens cirurgicamente acometidas, comprometendo a radicalidade do procedimento e o prognóstico da doença. Não se observou diferença entre os tumores mucinosos e não-mucinosos quanto à invasão de veias e nervos, o que pode ser devido à destruição do tecido pelo tumor, impedindo o exame desse parâmetro.

Adenomas preexistentes no local do CCR associam-se a doença menos avançada ou agressiva. Neste grupo observamos que o CCR se infiltrava menos profundamente na parede do órgão, com menor acometimento vascular e de nervos, embora o acometimento de vasos linfáticos fosse semelhante aos demais tumores. A literatura relata a associação de adenomas, principalmente o do tipo viloso, com o adenocarcinoma ${ }^{(4,14,16,19,27)}$. Loy et al. ${ }^{(15)}$ demonstraram correlação entre a presença de pólipos adenomatoso, viloso ou tubuloviloso com o CCR. A presença do adenoma foi inversamente proporcional ao estadiamento do tumor e à sobrevida. Uma justificativa possível é a destruição do adenoma pelo tumor em crescimento. Os adenomas também foram mais comumente encontrados associados a tumores sincrônicos.

A detecção de tumores sincrônicos é importante porque eles são descritos como mais agressivos e apresentando metástases a distância mais precocemente ${ }^{(21,30)}$, o que não foi observado em nosso estudo. Este grupo de tumores, assim como a presença de adenoma, está comumente relacionado com síndromes genéticas ${ }^{(4,16,27)}$, sendo a história familiar um importante fator de estudo, além da necessidade da pesquisa de tumores sincrônicos durante a propedêutica dos pacientes.

\section{Conclusões}

Os dados de nossa casuística de CCR não diferiram do que foi relatado na literatura para a nossa população. Em síntese, o CCR foi mais comum em mulheres do que em homens, acometeu com maior freqüência o cólon esquerdo, principalmente o reto, e o tipo predominante foi o adenocarcinoma moderadamente diferenciado, pT3 e pN0. Não se observou diferença quanto ao estadiamento em relação a sexo, idade ou localização do tumor. Em cerca de $10 \%$ dos casos os pacientes tinham menos de 40 anos $(10,7 \%)$, sendo candidatos a testes genéticos para determinação do caráter hereditário da doença. No nosso meio, o CCR foi uma doença diagnosticada em fases avançadas, diminuindo a possibilidade de cura e, portanto, de prognóstico mais reservado.

\section{Referências}

I.ASTHER,V. B.; COLLER, F.A.The classification significance of direct extension of carcinoma of the colon and rectum. Annals of Surgery, v. 139, n. 6, p. 846-5।, 1954.

2. BALDUS, S. E. et al. Comparative evaluation of the prognostic value of MUCI, MUC2, sialyl-Lewisa and sialyl-Lewisx antigens in colorectal adenocarcinoma. Histopathology, v. 40, p. 440-90, 2002.
3. BIEMER-HÜTTMANN,A-E. et al. Mucin core protein expression in colorectal cancer with high leves of microsatellite instability indicates a novel pathway of morphogenesis. Clinical Cancer Research, v. 6, p. 1909-16. 2000.

4. BODMER, W. F.; WILDING, J.; FEARNHEAD, N. S. Molecular genetics of colorectal cancer. Acta Oncologica Brasileira, v. I, n. 24, 2004. Disponível em: <http://www.hcanc.org.br/ 
acta/2004/acta04_07.html>.Acesso em: I 6 nov. 2004.

5. BOSMAN, F.T. Prognostic value or pathological characteristics of colorectal cancer. Eur J Cancer, v. 3। A, p. 12 16-2 I, 1995.

6. DRUMOND, C. A. et al. Câncer colorretal em pacientes com idade inferior a 30 anos. Rev Bras Coloproct, v. 23, n. 3, p. |47-54, 2003.

7. DUARTE, A. P. et al. Importância da invasão neural e linfática no prognóstico do adenocarcinoma colorretal. Rev Assoc Med Bras, v. 50, n. I, p. 21-6, 2004.

8. DUKES, C. E. The classification or cancer of the rectum.J Pathol Bact, v. 35, p. 323-32, 1932.

9. GARFINKEL, L; MUSHINSKI, M. U.S. cancer incidence, mortality and survival: 1973-1996. Stat Bull Metrop Insur Co, v. 80, p. 23-32, 1999.

10. GERVAZ, P. et al. Dukes B colorectal cancer: distinct categories and clinical outcome based on proximal or distal tumor location. Dis Colon Rectum, v. 44, n. 3, p. 364-73, 2001.

I I. HARMILTON, S. R. et al. Tumours of the colon and rectum. In: HARMILTON, S. R.; AALTONEN, L. A. World Halt Organization Classification of the Tumors. Pathology \& Genetics. Tumors or the digestive system. I. ed. Lyon: IARC Press, 2000. cap. 6, p. 103-42.

12. INSTITUTO NACIONAL DO CÂNCER. Estimativas da incidência e mortalidade por câncer no Brasil 2003. Disponível em: <http:// wnw.inca.gov.Br>. Acesso em: 9 jul. 2004.

13. KOYAMA, Y.; KOTAKE, K. Overview of colorectal cancer in Japan: report from the registry of the Japanese Society for Cancer of the Colon and Rectum. Dis Colon Rectum, n. 40, v. 10, p. S2-9, 1997.

14. KUBOTA, D. et al. Nonpolypoid adenomas and adenocarcinomas foud in background mucosa of surgically resected colons. Cancer, v. 4, n. 77, p. 621-6, 1996.

15. LOY,T. S.; KAPLAN, P. A. Villous adenocarcinoma of the colon and rectum. Am J Pathol, v. 28, p. 1460-5, 2004.

16. LYNCH, H.T. HNPCC (Lynch syndrome): differential diagnosis, molecular genetics, surveillance and management. Acta Oncológica Brasileira, v. I , n. 24, 2004. Disponível em: <http:// www.hcanc.org.br/acta/2004/acta04_06.html>.Acesso em: 16 nov. 2004.

17. MENDES, M. B. P.; COSTA, L. D. B.; TAMURA, S. Tumores do cólon e reto: experiência de 16 anos. Rev Bras Coloproct, v. 12, n. 4, 1992.
18. MESSERINI, L. et al. Prognostic significance of microsatellite instability in sporadic mucinous colorectal cancers. Hum Pathol, v. 6, n. 30, p. 629-34, 1999.

19. MINAMOTO, T. et al. Superficial-type adenoma and adenocarcinoma of the colon and rectum: a comparative morphological study. Gastroenterology, v. 106, p. 1436-43, 1994.

20. NEWLAND, R. C. et al. Pathologic determinants or survival associated with colorectal cancer with lymph node metastases: a multivariate analysis of 579 patients. Cancer, v. 73, p. 2076-82, 1994

21. OYA, M. et al. Synchronous colorectal carcinoma: clinicopathlogical features and prognosis. I Clin Oncol, v. 33, n. I, p. 38-43, 2003.

22. PETERSEN, G. M. Genetic epidemiology of colorectal cancer. European Journal of Cancer, v. 31, n. A, p. 1047-50, 1995.

23. PHILLIPS, R. K. S. et al. Large bowel cancer: surgical pathology and its relationship to survival. Br J Surg, v. 71, p. 604-10, 1984.

24.PINHO, M. Câncer colorretal com instabilidade de microssatélites: uma doença diferente. Rev Bras Coloproct, v. 22, n. 2, p. 139 44, 2002.

25. PONK DE LEON, M. et al.Trend of incidence, subsite distribution and staging of colorectal neoplasms in the 15-year experience of a specialized cancer registry. Ann Oncol, v. I5, n. 6, p. 940-6, 2004.

26. POTTER, J. D. Colorectal cancer: molecules and populations. J Natl Cancer Inst, v. 91, p. 916-32, 1999.

27. ROSSI, B. M. et al. Câncer colorretal hereditário sem polipose: HNPCC. Acta Oncológica Brasileira, v. I, n. 22, 2002. Disponível em: URL: <http://www.hcanc.org.br/acta/2002/ acta02_4.html>. Acesso em: 16 nov. 2004.

28. SALLES, P. G. O.; NOGUEIRA, A. M. M. F. Câncer colorretal: preparo e análise de peças cirúrgicas. In: CASTRO, L. P. Tópicos em gastroenterologia I I: avanços em coloproctologia. I. ed. Rio de Janeiro: Editora Médica e Científica Ltda., 200 I. cap. 13, p. 167-74.

29.SOBIN, L. H.;WITTEKIND, C. H. Colon and rectum. In:SOBIN, L. H.;WITTEKIND, C. H. TNM classification of malignant tumors. 6. ed. New York:Willy-Liss, 2002. p. 72-6.

30. WANG, H. Z. Clinical features, diagnosis, treatment and prognosis of multiple primary colorectal carcinoma. World J Gastroenterology, v. I0, n. 14, p. 2136-9, 2004 
Anexo - Protocolo de exame anatomopatológico para carcinoma colorretal utilizado no serviço.

\section{CARCINOMAS DE CÓLON E RETO}

\section{Procedimento cirúrgico:}
( ) colectomia segmentar
( ) enterocolectomia
( ) retocolectomia
( ) amputação de reto

( ) com (outro órgão)

\section{EXAME MACROSCÓPICO}

\section{Peça cirúrgica}

Comprimento............cm;

perímetro distal.............cm

perímetro proximal.

(médio. ..cm)

\section{Localização do tumor}
( ) ceco
( ) cólon transverso
( ) cólon sigmóide
( ) cólon ascendente
( ) flexura esplênica
( ) grande curvatura
( ) flexura hepática
( ) cólon descendente
( ) reto

\section{Pólipo preexistente (no local do carcinoma)}

( ) não há evidências de pólipo preexistente

( ) há evidência de pólipo preexistente do tipo

Tamanho do tumor ( $\mathrm{L} \times \mathrm{T} \times \mathrm{P})$ : $\mathrm{X}$ $\mathrm{X}$ $\mathrm{cm}$ Aspecto macroscópico
( ) polipóide
( ) anular-constritivo
( ) ulceroinfiltrativo
( ) difuso
( ) elevado tipo placa
( ) outro

\section{Perfuração tumoral}
( ) presente
( ) ausente
( ) não-identificada

\section{Margens de ressecção:}

Proximal

( ) livre $(\ldots \ldots . . . . \mathrm{cm})$

( ) envolvida

Distal

( ) livre $(\ldots \ldots . . . \mathrm{cm})$

( ) envolvida

Radial

( ) livre $(\ldots \ldots . . . \mathrm{cm})$

( ) envolvida

(margem de tecidos moles mais proximais ao ponto de máxima penetração tumoral)
Margens de segurança (disco)
( ) não se aplica $\mathrm{cm}$

\section{Invasão do meso-reto distal}
( ) não se aplica
( ) presente
( ) ausente
( ) não-visível

\section{Comprometimento da superfície serosa (estimado)}
( ) presente
( ) ausente
( ) não-visível

Linfonodos (total):

Peritumorais: (..............)

Distais TU: (...)
) número de linfonodos

proximais TU (até $5 \mathrm{~cm}$ do $\mathrm{TU})$ : (..............) restantes: (..............) 


\section{EXAME MICROSCÓPICO}

\section{Tipo de neoplasia (OMS)}
( ) adenocarcinoma SOE
( ) Ca mucinoso (colóide)
( ) Ca de células escamosas
( ) Ca de células em anel de sinete
( ) Ca basalóide (cloacogênico)
( ) Ca adenoescamoso
( ) Ca indiferenciado de pequenas células
( ) sem tumor residual

Grau de diferenciação
( ) bem diferenciado
( ) moderadamente diferenciado
( ) pouco diferenciado
( ) indiferenciado

$\begin{array}{lllll}\text { Invasão vascular sangüinea } & \text { ( ) presente } & \text { ( ) ausente } & \text { ( ) não-avaliável } & \\ \text { Invasão vascular linfática } & \text { ( ) presente } & \text { ( ) ausente } & \text { ( ) não-avaliável } & \\ \text { Invasão perineural } & \text { ( ) presente } & \text { ( ) ausente } & \text { ( ) não-avaliável } & \\ \text { Resposta linfocítica peritumoral } & \text { ( ) ausente } & \text { ( ) discreta } & \text { ( ) moderada } & \text { ( ) acentuada }\end{array}$

Invasão do tumor (pT):

( ) carcinoma in situ/restrito à lâmina própria - pTis

( ) invasão da submucosa - pT1

( ) invasão da muscular própria - pT2

( ) invasão da subserosa/tecidos moles pericólicos ou peri-retais - pT3

( ) invasão do peritônio visceral ou invasão direta de (órgãos/estruturas adjacentes) - pT4

\section{Margem microscópica}
( ) não se aplica
( ) livre ( $\mathrm{cm})$
( ) envolvida

\section{Margens de segurança (disco)}
( ) não se aplica
( ) livre ( $\mathrm{cm})$
( ) envolvida

\section{Linfonodos (pericólicos/peri-retais):}

Peritumorais com metástases: sem metástases:

Proximais ao TU com metástases: sem metástases:

Distais ao TU com metástases: sem metástases:

Restantes com metástases:

Em correspondência c/..... sem metástases: com metástases:..... sem metástases: outros: com metástases:. sem metástases:

\section{Estadiamento dos linfonodos (pN)}
( ) $\mathrm{pNx}$ (não-avaliável) (menos de 12 linfonodos dissecados)
( ) pNO - não se observam metástases em linfonodos regionais
( ) pN1 - metástases em ....... linfonodos pericólicos/peri-retais (1-3)
( ) pN2 - metástases em ........ linfonodos pericolicos/peri-retais (4 ou mais)
( ) pN3 - metástases em ....... linfonodos ao longo da (tronco vascular específico) (1 ou mais)

Intestino não-neoplásico: ( ) sem anormalidades
( ) pólipos isolados: .(número) tipo
( ) divertículos
( ) polipose familiar
( ) retocolite ulcerativa,
( ) com
( ) sem displasia
( ) esquistossomose
( ) outras

\section{Estadiamento patológico}

TNM: pT $\quad \mathrm{pN}$

(Astler-Coller: A - Restrito A Mucosa.; B1 - submucosa ou musc. própria - pN0 - pM0; B2 - serosa - pN0 - pM0; C1 - submucosa/musc. própria - pN1 ou >, pM0; C2 - serosa qq pN - pM0; D - qq pT, qq pN, pM1)

(Dukes: A - pT1 OU pT2, pN0, pM0; B - - pT3 OU pT4, pN0, pM0; C - qualquer pT até pN3; D - qualquer pT e pN. M1)

\section{Conclusão:}

